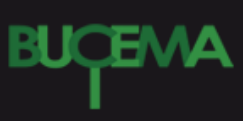

Bulletin du centre d'études médiévales d'Auxerre | BUCEMA

Hors-série $n^{\circ} 3 \mid 2010$

Présentation et mise en valeur des sites archéologiques religieux en milieu urbain

\title{
Les vestiges du premier groupe cathédral de Grenoble et leur présentation muséographique
}

Alain de Montjoye

\section{(2) OpenEdition}

Journals

Édition électronique

URL : https://journals.openedition.org/cem/11356

DOI : $10.4000 /$ cem. 11356

ISSN : 1954-3093

Éditeur

Centre d'études médiévales Saint-Germain d'Auxerre

Référence électronique

Alain de Montjoye, « Les vestiges du premier groupe cathédral de Grenoble et leur présentation muséographique ", Bulletin du centre d'études médiévales d'Auxerre | BUCEMA [En ligne], Hors-série n 3 | 2010, mis en ligne le 16 février 2010, consulté le 04 mars 2023. URL : http://journals.openedition.org/ cem/11356 ; DOI : https://doi.org/10.4000/cem.11356

Ce document a été généré automatiquement le 4 mars 2023.

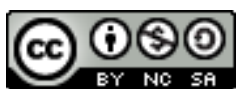

Creative Commons - Attribution - Pas d'Utilisation Commerciale - Partage dans les Mêmes Conditions 4.0 International - CC BY-NC-SA 4.0

https://creativecommons.org/licenses/by-nc-sa/4.0/ 


\title{
Les vestiges du premier groupe cathédral de Grenoble et leur présentation muséographique
}

\author{
Alain de Montjoye
}

Quand fut envisagée, vers la fin des années 1980, la réalisation à Grenoble d'une nouvelle ligne de tramway empruntant la place Notre-Dame et passant au pied de la cathédrale, il n'a pu être ignoré le fort impact archéologique qu'aurait cet aménagement. On savait, en effet, qu'on y rencontrerait l'enceinte urbaine du BasEmpire (fig. 1) et les substructions de l'une des deux portes monumentales qui s'y ouvraient : la porte Viennoise qui, jusqu'en 1802, avait fermé la place du côté nord. Ce sont également des parties importantes de l'ancien palais épiscopal (fig. 2), disparues en même temps que la porte, que les travaux n'allaient pas manquer d'exhumer et, bien sûr, ceux probables d'installations plus anciennes, appartenant au complexe cathédral primitif que l'on avait de bonnes raisons de croire fixé à cet emplacement dès sa création vers la fin du Iv siècle. 


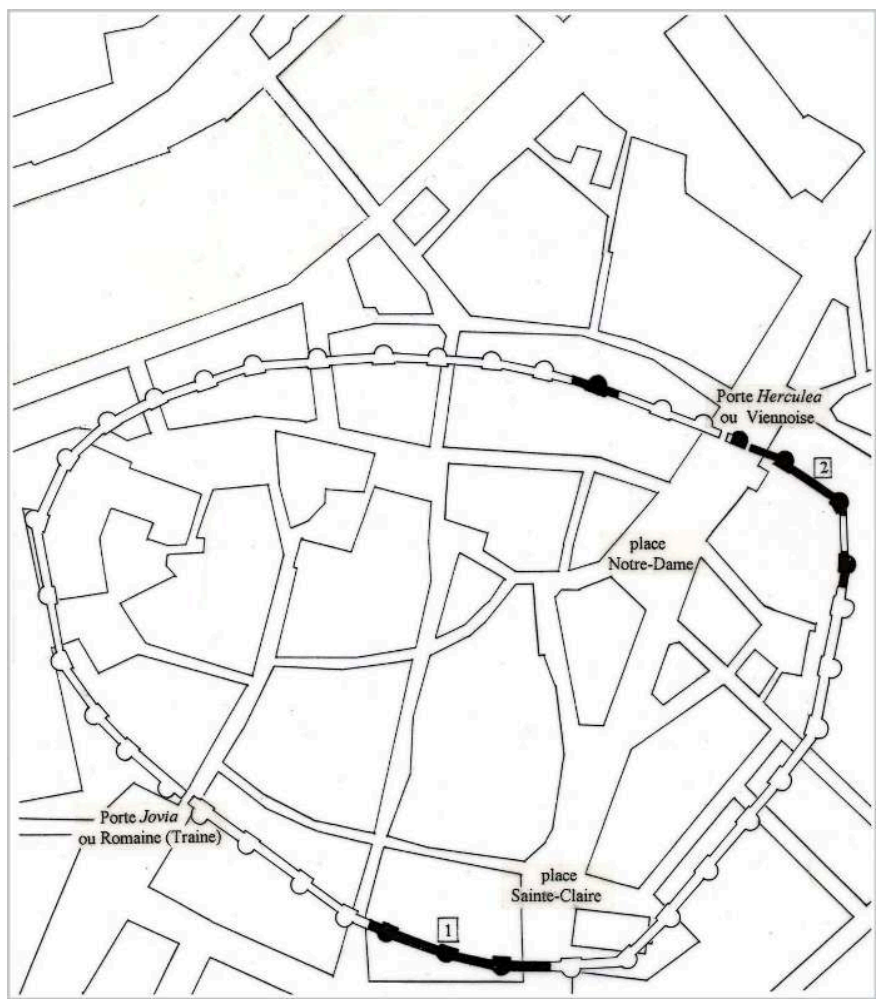

Fig. 1 - Tracé restitué de l'enceinte antique de Grenoble (en noir, les tronçons récemment observés et relevés).

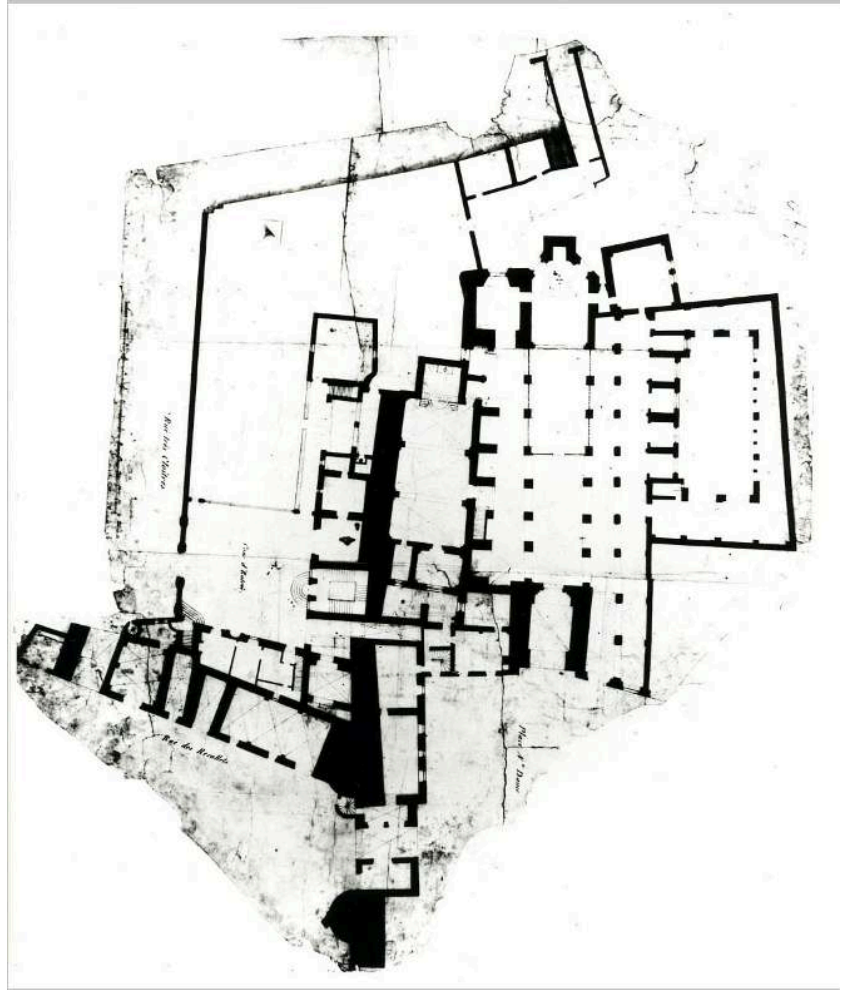

Fig. 2 - L'ensemble cathédral à la fin du XVIIle siècle (Arch. Dép. Isère 1 Fi 970).

2 Les fouilles archéologiques se sont insérées dans le déroulement d'un chantier complexe de grande ampleur qui a vu, simultanément, la mise en place de la voie de tramway et la construction d'un espace hypogée protégeant les vestiges 
archéologiques, par le soin des services de la Ville de Grenoble ; la restauration, par le service des Monuments historiques, des façades de la cathédrale médiévale, après dépose de la façade postiche derrière laquelle elles avaient été dissimulées à la fin du $\mathrm{XIX}^{\mathrm{e}}$ siècle; enfin, une reprise complète des bâtiments de l'ancien palais épiscopal et leur transformation en musée, sous l'égide du Département de l'Isère. Trois maîtrises d'ouvrage donc et autant de maîtrises d'œuvre, pendant sept années (1989-1995), se sont activées conjointement sur le même site.

Les fouilles archéologiques induites par ces divers aménagements ont elles-mêmes été réalisées par deux équipes travaillant côte à côte. L'une d'elles, relevant de l'AFAN et dirigée par François Baucheron, aujourd'hui responsable d'opérations à l'INRAP, s'est attachée au dégagement et à l'étude des vestiges découverts sous la place Notre-Dame, lesquels constituent la part majeure de ce qui est désormais visible et présenté dans l'espace hypogée créé à cet effet. L'autre équipe, sous la responsabilité de l'auteur de ces lignes, a travaillé dans l'emprise de l'ancien palais et autour de celle-ci, c'est à dire dans les limites de la propriété du Département de l'Isère dans les services duquel il est agent (fig. 3). La découverte et l'identification, dans les premiers mois de la fouille, en 1989, des vestiges d'un baptistère ont constitué l'événement majeur qui a conduit à modifier en cours de chantier le tracé de la voie ferrée, pour préserver les précieux restes et en permettre l'accès au public. Sous une dalle conçue pour permettre cet accès, les études archéologiques se sont poursuivies, cette fois en fouille programmée.

On ne commentera ici des résultats de sept années consécutives de fouilles ${ }^{1}$ que ce qui est nécessaire à l'appréhension des choix qui ont présidé - la part étant faite des contraintes matérielles et techniques - à la conception de l'espace de présentation des vestiges et au traitement de ceux-ci.

5 Parmi les apports les plus significatifs fournis par le site, dans l'espace compris sous la dalle, doivent être mentionnés ceux qui concernent la haute Antiquité, sous la forme de restes ténus de bâtiments dont le plus ancien pourrait remonter au II ${ }^{\mathrm{e}}$ siècle av. J.-C. D'autres, datables des $\mathrm{I}^{\mathrm{er}}$ et $\mathrm{II}^{\mathrm{e}}$ siècles de notre ère ou plus tardifs encore, montrent une occupation continue de l'endroit avant l'érection de l'enceinte urbaine de la fin du III ${ }^{\mathrm{e}}$ siècle. Une occupation qui excédait (sans que l'on puisse évaluer sur quelle distance) la limite introduite par l'enceinte, à l'extérieur de laquelle certaines constructions furent abandonnées (fig. 4). 


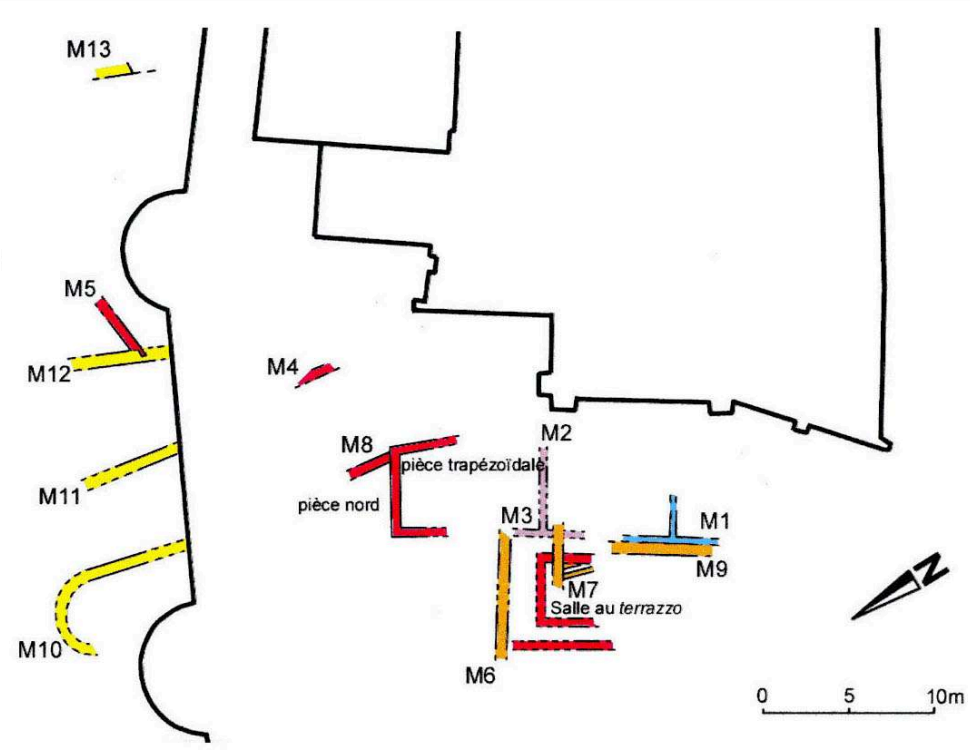

\section{Vestiges antiques}
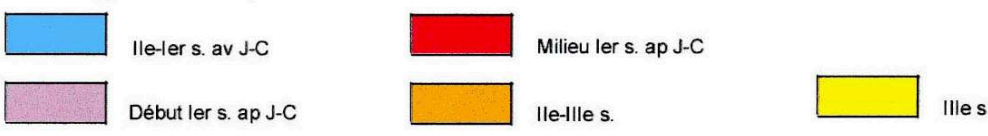

Fig. 4 - Vestiges de bâtiments antérieurs à la construction de l'enceinte (fin du Ille siècle).

Concernant l'enceinte elle-même, avec le dégagement sous le sol de la place d'un tronçon attenant à l'ancienne porte Viennoise et le prolongement de celui-ci sous l'ancien palais épiscopal, c'est quelques $30 \mathrm{~m}$ de cet ouvrage, conservé sur environ 2,50 $\mathrm{m}$ de hauteur en moyenne, incluant une tour en élévation et les substructions de celle qui flanquait au nord l'ancienne porte, qui apparaissent dans l'espace aménagé (fig. 5). Quantité d'informations ont été fournies par la fouille sur les techniques de construction : mode de mise en place des fondations (par endroits coulées en fosse, en d'autres élevées en tranchée large), échafaudages, parements, etc. Le tracé de l'escarpe du fossé a pu, sur ce linéaire, être restitué. 


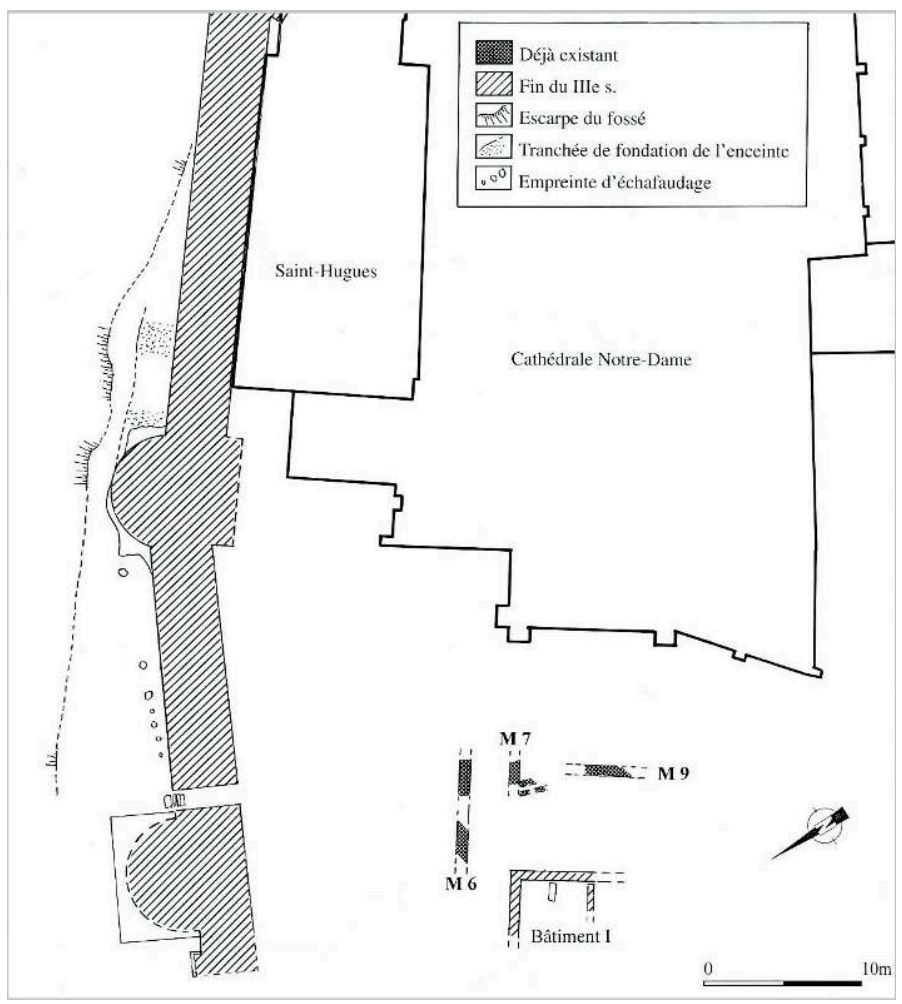

Fig. 5 : Les travaux de fortification de la fin du IIIe siècle.

7 À partir du IV siècle, prennent place contre la face interne du mur d'enceinte, des bâtiments dessinant un plan en L. S'il ne peut être affirmé que ces bâtiments sont, dès l'origine, liés au groupe épiscopal, ils y sont assurément intégrés, avec de premières modifications datables de la fin du Iv ${ }^{\mathrm{e}}$ siècle.

D'après les données recueillies en fouille, ce n'est pas avant le ve siècle qu'il convient de dater le baptistère dans sa forme la plus ancienne connue : une simple salle de plan carré, pourvue d'une cuve octogonale en son centre (fig. 6). La construction de ce baptistère s'accompagne d'une reconstruction sur un plan plus large de l'aile ouest du $\mathrm{IV}^{\mathrm{e}}$ siècle, dans laquelle il s'insère. Sans pouvoir toujours réunir des arguments de datation très précis, l'approche archéologique a mis en évidence un long processus de modifications, agrandissement et embellissement du baptistère lui-même qui, doté dans un premier temps d'une abside sur sa face est, finit par présenter une forme tétraconque. Dans le même temps, la cuve connaît des réductions successives, traduisant l'évolution rituelle que constitue le passage du baptême par immersion au baptême par infusion, jusqu'à présenter une forme pentagonale, pour le moins inhabituelle. Ces diverses transformations s'accompagnent de modifications des systèmes d'adduction et d'évacuation de l'eau (fig. 7). 


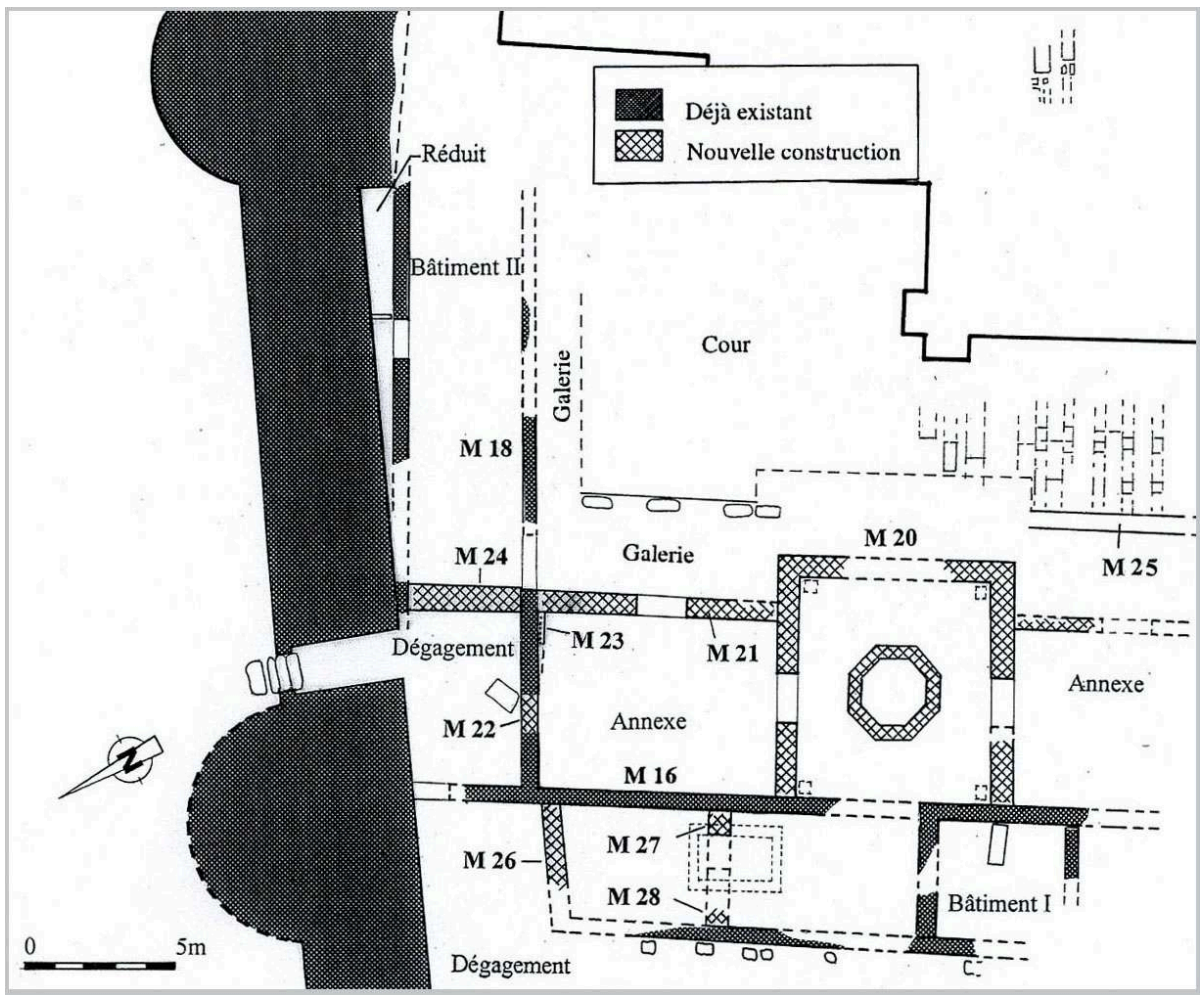

Fig. 6 - Ensemble des bâtiments après construction du baptistère au Ve siècle.

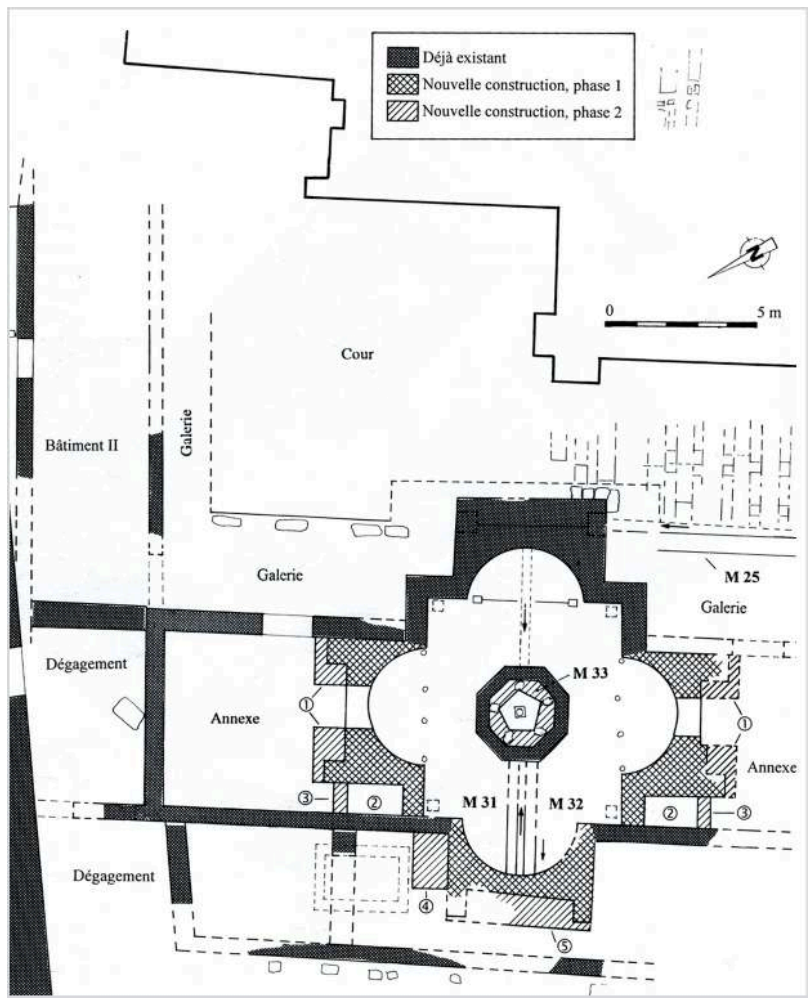

Fig. 7 - Les bâtiments tels qu'ils subsistaient encore à la période carolingienne.

9 Riche d'enseignements et plus encore source de questionnements, la présence de plusieurs sépultures regroupées sur l'étroite bande de terre ménagée entre le rempart et son fossé a constitué une véritable surprise (fig. 8). Avec des incertitudes quant à la 
datation - de forts indices militent pour la fin du $\mathrm{IV}^{\mathrm{e}}$ siècle - et des arguments pour interpréter cette aire d'inhumation comme liée à un épisode de catastrophe, on ne peut s'empêcher de penser que le choix de son emplacement - hors les murs, sans doute, conformément à l'usage antique - n'est pas sans rapport avec le voisinage immédiat, de l'autre côté du mur, du complexe cathédral.

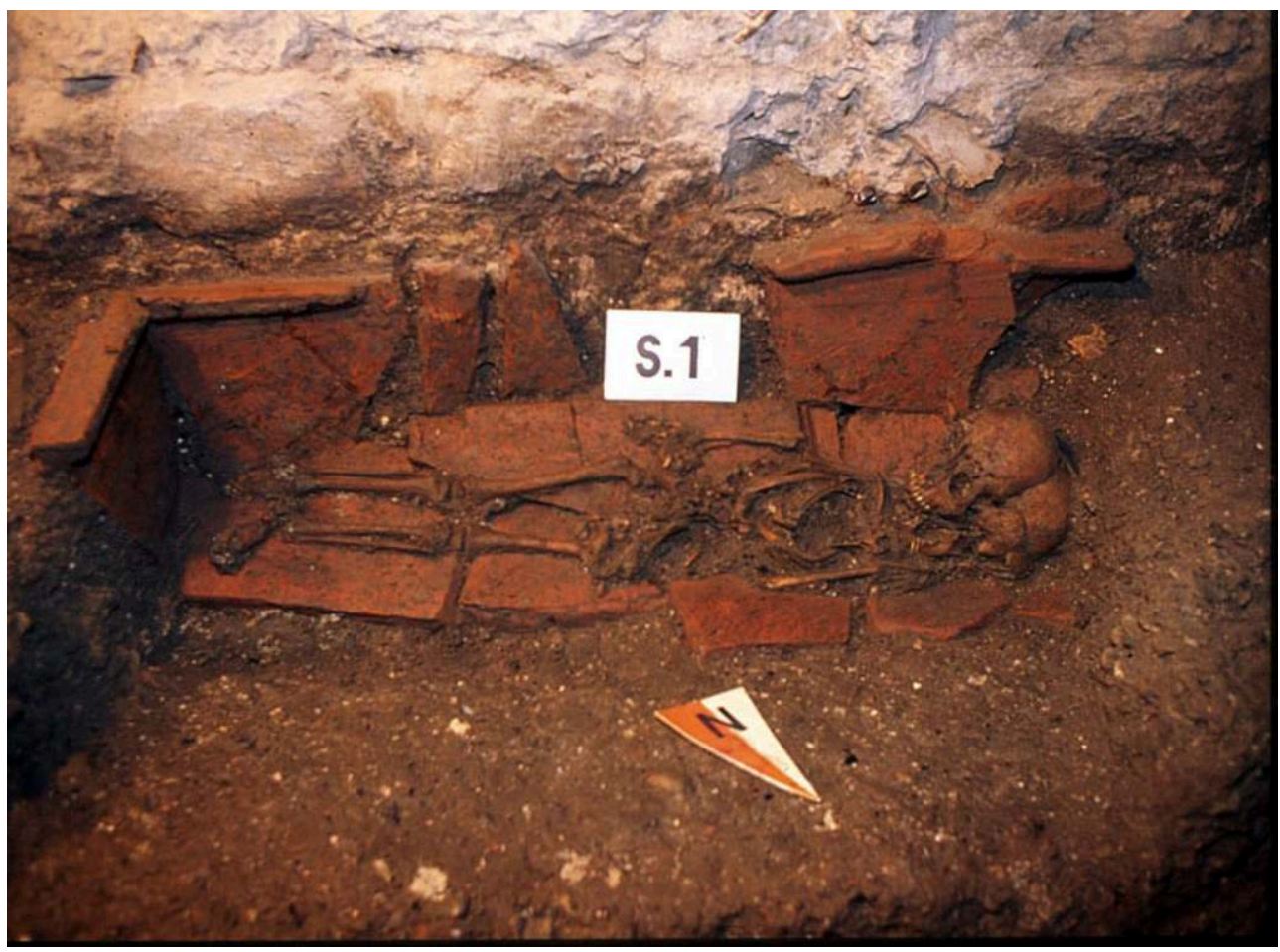

Fig. 8 - Sépulture d'enfant en coffre de tegulae.

10 Un complexe cathédral dont il est possible, sinon de fournir une restitution précise et fiable, au moins de proposer un aperçu schématique : deux probables églises disposées côte à côte, précédées d'une cour pavée (atrium) fermée par des bâtiments que longent des portiques. Le baptistère se dresse au centre du corps de bâtiment ouest (fig. 9). 


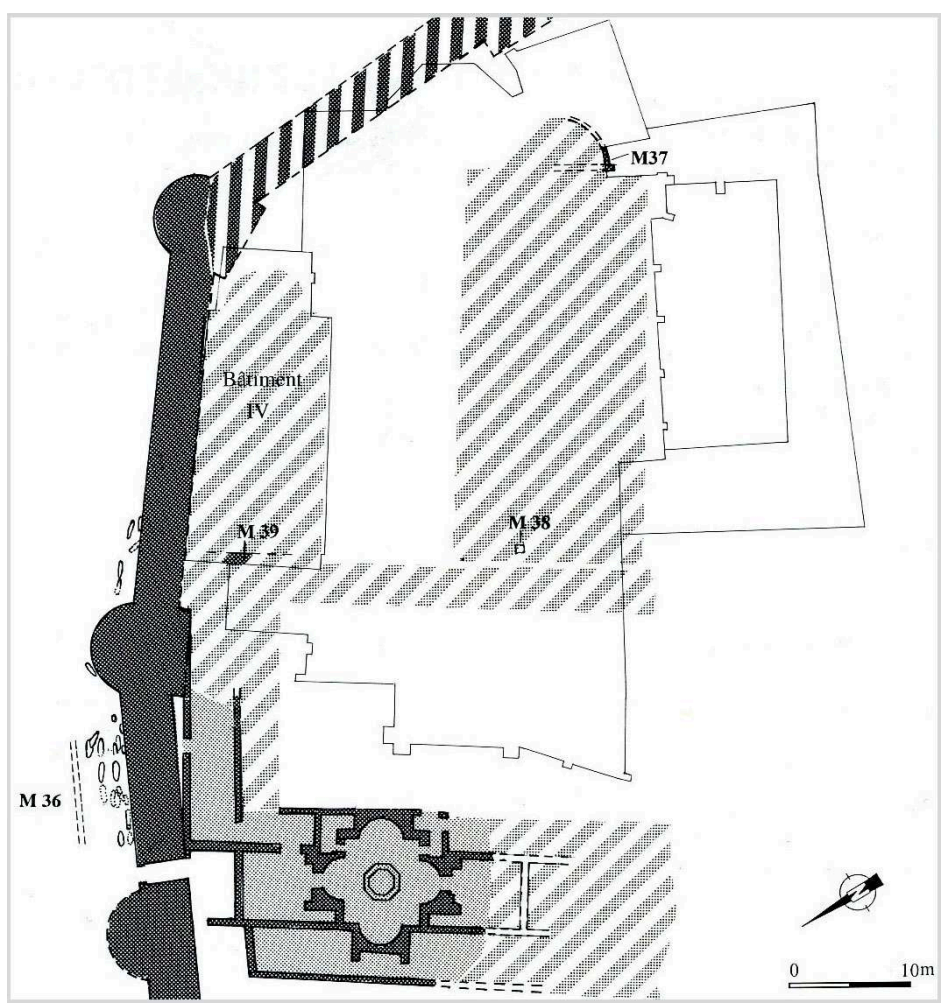

Fig. 9 - Restitution schématique du groupe cathédral du haut Moyen Âge.

11 Avec les évolutions précédemment signalées, cet ensemble est resté en fonction jusque dans la période carolingienne. Dès la fin de cette période, des destructions partielles et de nouveaux bâtiments de conception assez grossière témoignent d'une désorganisation progressive du complexe architectural hérité des premiers temps chrétiens.

Enfin, peut-être dès le $\mathrm{x}^{\mathrm{e}}$ siècle, assurément au cours $\mathrm{du} \mathrm{XI}^{\mathrm{e}}$ et des suivants, la multiplication de sépultures à l'emplacement des bâtiments marque l'abolition des dispositions architecturales premières et l'irruption des morts dans l'espace urbanisé. Le cimetière paroissial ne disparaîtra de cet emplacement qu'à la fin du XvII ${ }^{\mathrm{e}}$ siècle.

Tenter de donner un aperçu le plus complet possible des riches informations recueillies sur le site était le défi que posait la réalisation d'un espace muséographique mettant en scène les vestiges. Disons d'emblée qu'il n'a été, de notre point de vue, que très imparfaitement relevé.

14 Il est vrai que le parti général d'aménagement du volume hypogée en deux secteurs séparés, parti absolument justifié et cohérent, a rendu immédiatement perceptible la distinction entre les deux espaces extra-muros et intra-muros, reliés par l'unique passage que constitue une ancienne poterne qui existait dès l'origine à gauche (est) de la porte monumentale. Là où le mur n'est conservé que sur une faible hauteur, un voile opaque a été réalisé qui en prolonge les parements jusqu'au plafond.

Sur le détail des réalisations, il nous faut, en revanche, exprimer plusieurs regrets. 


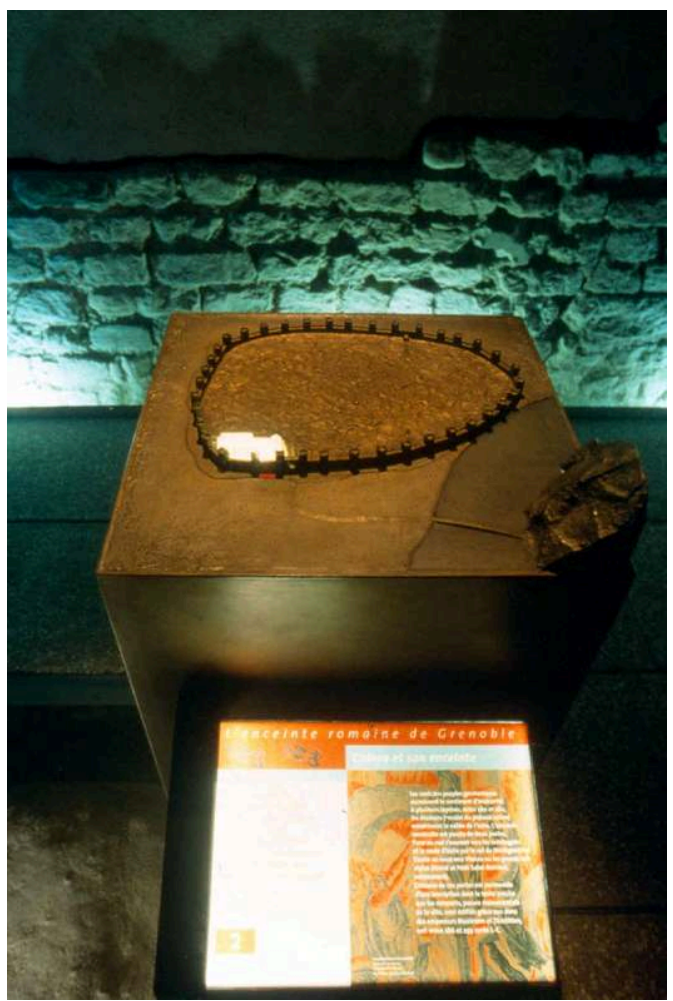

Fig. 10 - maquette représentant Grenoble à la fin du IVe siècle.

16 L'espace extra-muros, qui se présente comme un étroit passage au pied du rempart, est meublé de divers socles et vitrines porteurs d'informations très générales sur la période gallo-romaine. Si l'on excepte une très utile maquette localisant le groupe cathédral à l'intérieur de l'enceinte antique (fig. 10), l'attention est portée sur l'étendue de l'ancienne cité de Vienne, une transposition de la Table de Peutinger, des évocations de la vie quotidienne et des cultes au moyen de divers objets, issus pour la plupart de collectes anciennes en tous points de la ville. En place de ces données qui peuvent être fournies tout aussi bien ailleurs, il s'imposait, selon nous, d'évoquer la problématique de la primitive étendue urbaine et de sa rétractation dans l'enceinte du ${ }{ }^{\mathrm{e}}{ }^{\mathrm{e}}$ siècle, de valoriser la compréhension nouvellement acquise de cette enceinte et de ses modes de construction, d'informer sur la petite nécropole paléochrétienne et les questions que sa présence suscite. Rien de tout cela, si ce n'est, au sol, une étroite lucarne de verre à l'aplomb de maçonneries antérieures au rempart, que la vision tronquée rend incompréhensibles (fig. 11). 


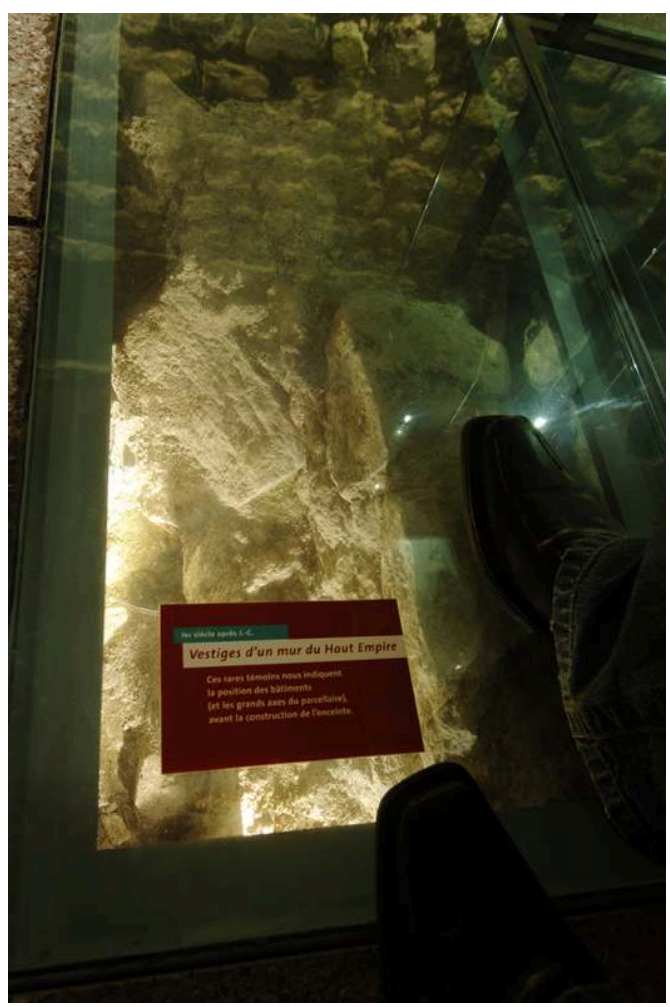

Fig. 11 - En ce point deux murs de directions et datations différentes se superposent. Les dimensions insuffisantes de la lucarne n'en permettent pas la lecture.

L'espace intra-muros, beaucoup plus vaste que le précédent, est voué, à l'exclusion de tout autre objet, à la mise en scène des vestiges du seul baptistère. Rien ne peut se percevoir (et encore moins être compris) des bâtiments au sein desquels le baptistère prenait place. Des surfaces de circulation en caillebotis, qui prétendent en reproduire le plan à l'aplomb, laissent à peine percevoir certains murs et seulement dans une vision strictement verticale. Nulle évocation des transformations que ces bâtiments ont connues, nulle vision de leurs sols en mortier de tuileau, aujourd'hui dissimulés sous des graviers dans lesquels courent les câbles d'alimentation électrique, sols pieusement laissés en place au détriment de l'information archéologique, en vue précisément de leur présentation!

Si trois maquettes et quelques éléments mobiliers trouvés dans la fouille facilitent utilement la compréhension des vestiges du baptistère, souci de simplification extrême et de clarté prévalant, on a jugé inopportun de donner à saisir quoi que ce soit des processus de désorganisation du complexe cathédral primitif, des constructions du haut Moyen Âge finissant, de l'implantation du cimetière médiéval. Sans doute l'exercice était-il périlleux de vouloir exprimer la complexité des faits archéologiques. Mon regret est qu'il n'ait pas été tenté ${ }^{2}$. Un regret d'autant plus sincère que la réalisation, extrêmement soignée et esthétique, ne pouvait qu'y gagner.

J'ajouterai, pour finir, qu'un musée de site aussi important et minutieusement conçu que celui du baptistère de Grenoble est un équipement coûteux, dont l'entretien réclame des soins constants ${ }^{3}$. Aménagé en sous-sol et donc exposé aux infiltrations, et aux remontées d'humidité, donc au développement de micro-organismes déprédateurs, l'atmosphère doit y être en permanence régulée. À Grenoble, une imposante machinerie a été conçue pour maintenir un équilibre hygrométrique et de température 
constant, en même temps qu'un renouvellement régulier de l'air. Néanmoins, les apparitions de mousses et de moisissures sont récurrentes (fig. 12). Sans compter d'imprévisibles accidents tels que des infiltrations en provenance d'un collecteur d'égout voisin fissuré.

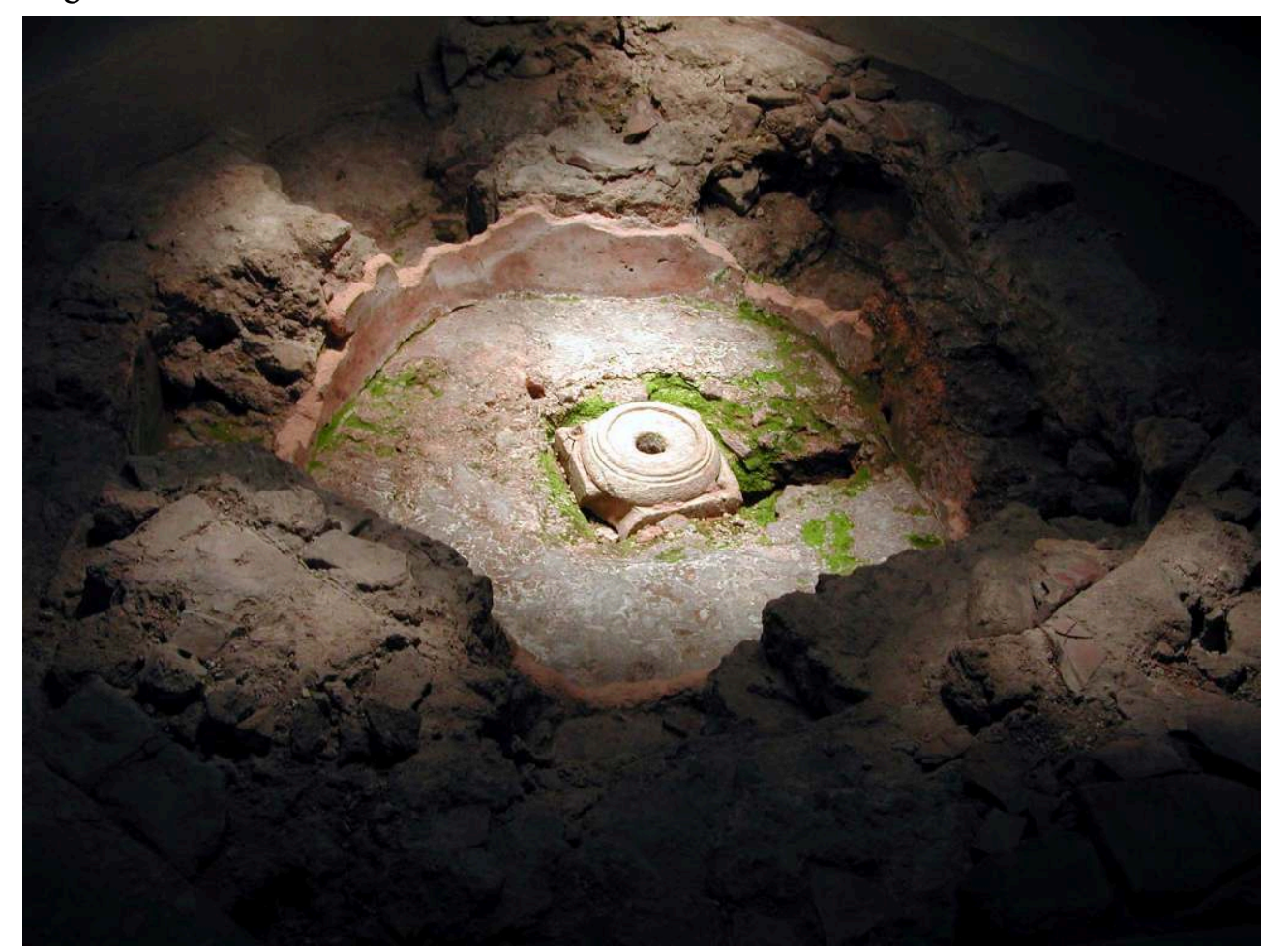

Fig. 12 - Formation de mousses dans le fond de la cuve du baptistère.

L'intérêt croissant manifesté par le public pour les vestiges du passé rendus par l'archéologie constitue, bien sûr, la justification suffisante des efforts engagés par la collectivité pour leur mise en valeur et leur entretien.

3. L'espace de présentation du baptistère est accessible depuis le musée de l'Ancien Evêché qui relève des services du Conseil général de l'Isère. Géré, entretenu et animé par le musée, il reste propriété de la Ville de Grenoble. Voir l'article de notre collègue Isabelle LAZIER, « La restauration du groupe évêché-cathédrale de Grenoble: un choix affirmé en faveur du patrimoine », à paraître dans Monumental (2009).

\section{NOTES}

1. Ils ont été publiés en 1998 : F. BAUCHERON, F. GABAYET, A. DE MONTJOYE, Autour du groupe épiscopal de Grenoble: deux millénaires d'histoire (Documents d'Archéologie en Rhône-Alpes $\left.n^{\circ} 16\right)$, Lyon.

2. L'incontestable réussite des aménagements de vestiges sous la cathédrale de Genève, procède de choix radicalement opposés à ceux retenus à Grenoble. 
INDEX

Index géographique : France/Grenoble

Mots-clés : groupe épiscopal 